\title{
Research on Aircraft Delay Ranking
}

\author{
Xiao Wang \\ School of North China Electricity Power University, Baoding 071000, China \\ 836269467@qq.com
}

Keywords: Cluster analysis, AHP

\begin{abstract}
In this paper, an improved AHP model based on clustering analysis is used to analyze the global main airports, and it gains the airport ranking which delay the most seriously. Compared with the project, results are not consistent with the results obtained from the project. Therefore it concludes that the outcome from project is not accurate. In the model, rating is based on the standard tier of different levels of delay, and according to the cluster analysis, it eliminates the airport agencies which deviated from the unified standard, and gets the weight of different levels of delay, and then calculates the total weighted delay rate to acquire the specific ranking.
\end{abstract}

\section{Data preprocessing}

For an airline, its sample size is the recorded flight volume which included actual takeoff, arrival or cancellation. Screening is according to the punctuality rate report of international airports ${ }^{[2]}$ around the world from June 2014 to March 2015, excluding sample size of international airport is relatively small. And then, select the sample size which is top 60 international large and medium-sized airport and to compare the punctuality rate in the next step.

\section{Calculate average delay rate}

The delay of airport flight is relevant with the month, and has a positive correlation to sample size of the airport showed from analysis. Therefore the average delay rates is the sum of monthly delay number in the airport divided the sum of monthly sample size. These two methods are used to reduce the impact of the specific month.

$$
P_{j}=\frac{\sum_{i=1}^{10} p_{i j} n_{i j}}{n_{i j}} \quad j=1,2 \cdots 60
$$

\section{Making models-Improved AHP model for cluster analysis}

\subsection{The principle of improved AHP method based on clustering analysis}

Applying analytic hierarchy process ${ }^{[4]}$ to determine the weight given by the airports, combining qualitative analysis with quantitative analysis, is to weakening the influence of subjective factors. By using cluster analysis ${ }^{[1]}$, and eliminating a certain or some agencies based on the standard of agencies or the degree of the standard deviation, and modifying the weights assigned by different airports , to make the option more reliable, it is that obtain the final weight between airports and evaluation index, and that provide the decision.

\subsection{Solving model :}

Decision making problem is divided into three tiers, the top tier is the target tier, that is the ranking of airport delay, the lowest tier is the project tier which is the screened $P_{1}, P_{2} \cdots P_{j}(j=1,2 \cdots 60) 60$ international airports. The middle tier is the standard tier which included three evaluation indexes composed of slightly delay, delay, and serious delay ${ }^{[3]}$.

The weight between the standard tier and target tier is determined by the correlation between standard tier(degree of delay) and the target tier(ranking of delay). According to the present data and selected 10 representative airport, by using the evaluation criteria 1-9 scale, the weight between 
any two of three delay degree can be calculated. The 10 comparison matrix is as follows:

$$
\begin{aligned}
& A_{1}=\left[\begin{array}{ccc}
1 & 1 / 3 & 1 / 5 \\
3 & 1 & 1 / 3 \\
5 & 3 & 1
\end{array}\right] A_{2}=\left[\begin{array}{ccc}
1 & 1 / 4 & 1 / 7 \\
4 & 1 & 1 / 3 \\
7 & 3 & 1
\end{array}\right] A_{3}=\left[\begin{array}{ccc}
1 & 1 / 3 & 1 / 6 \\
3 & 1 & 1 / 4 \\
6 & 4 & 1
\end{array}\right] A_{4}=\left[\begin{array}{ccc}
1 & 1 / 3 & 1 / 7 \\
3 & 1 & 1 / 4 \\
7 & 4 & 1
\end{array}\right] \quad A_{5}=\left[\begin{array}{ccc}
1 & 1 / 2 & 1 / 5 \\
2 & 1 & 1 / 2 \\
5 & 2 & 1
\end{array}\right] A_{6}=\left[\begin{array}{ccc}
1 & 1 / 4 & 1 / 6 \\
4 & 1 & 1 / 3 \\
6 & 3 & 1
\end{array}\right] \\
& A_{7}=\left[\begin{array}{ccc}
1 & 1 & 1 / 7 \\
1 & 1 & 1 / 5 \\
7 & 5 & 1
\end{array}\right] \quad A_{8}=\left[\begin{array}{ccc}
1 & 1 / 3 & 1 / 6 \\
3 & 1 & 1 \\
6 & 1 & 1
\end{array}\right] \quad A_{9}=\left[\begin{array}{ccc}
1 & 1 / 4 & 1 / 5 \\
4 & 1 & 1 / 3 \\
5 & 3 & 1
\end{array}\right] \quad A_{10}=\left[\begin{array}{ccc}
1 & 1 / 3 & 1 / 9 \\
3 & 1 & 1 / 6 \\
9 & 6 & 1
\end{array}\right]
\end{aligned}
$$

Calculation of weight vector and consistency check:

$$
A w=\lambda w
$$

Solving maximum eigenvalue $\lambda_{\max }$ of comparison matrix $A$, according to the formula (2) and the maximum eigenvalue to calculate the corresponding eigenvector $w$, and then standardizing $w$ which is the weight between a certain tier and any factors in different tier, based on the size of this weight, it can determine the sort of the factors.

$$
\begin{aligned}
& C I=\frac{\lambda-n}{n-1} \\
& C R=\frac{C I}{R I}<0.1
\end{aligned}
$$

\begin{tabular}{|c|c|c|c|}
\hline WeighnDDelay degree & 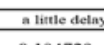 & Delhy & Serioum delayy- \\
\hline$w_{i}$ & 0.104729 & 0.258285 & 0.636986 \\
\hline$w_{2}$ & 0.078617 & 0.262753 & 0.658630 \\
\hline w, & 0.091402 & 0.217638 & 0.690059 \\
\hline w. & 0.084144 & 0.210920 & 0.704936 \\
\hline$w_{s}$ & 0.128271 & 0.276350 & 0.595379 \\
\hline$w_{\circ}$ & 0.085220 & 0.270557 & 0.644223 \\
\hline$w$ & 0.108472 & 0.261434 & 0.630093 \\
\hline$w_{*}$ & 0.104800 & 0.396120 & 0.49908 \\
\hline$w_{0}$ & 0.101323 & 0.255317 & 0.64360 \\
\hline$w_{10}$ & 0.067933 & 0.161755 & 0.770312 \\
\hline
\end{tabular}

Get the consistence $C I$ ( $n$ for $A$ 's dimension ), and random index $R I$, to check whether it is consistent with the formula (4), otherwise to adjust the comparison matrix until to get a satisfactory consistency.

The weight coefficients of the ten airports are obtained through calculation:

Set the size of weight aggregation about 10 airports and the weight coefficient is slightly delay, delay and serious delay. Solve the weight aggregation of evaluation index by analytic hierarchy process. $A=\left(a_{i j}\right)(i=1,2 \cdots, 10 ; j=1,2,3), \quad a_{i j}$ is the number $i_{\text {airport }}$ for the number $j$ factor's weight, and satisfied $\sum_{i=1}^{3} a_{i j}=1(j=1,2 \cdots 10)$.

The weight matrix which is processed by the analytic hierarchy process is as follows:

$$
A=\left[\begin{array}{ccc}
0.104729 & 0.258285 & 0.636986 \\
0.078617 & 0.262753 & 0.65863 \\
0.091402 & 0.217638 & 0.690959 \\
0.084144 & 0.21092 & 0.704936 \\
0.128271 & 0.27635 & 0.595379 \\
0.08522 & 0.270557 & 0.644223 \\
0.108472 & 0.261434 & 0.630093 \\
0.1048 & 0.39612 & 0.49908 \\
0.101323 & 0.255317 & 0.64336 \\
0.067933 & 0.161755 & 0.770312
\end{array}\right]
$$

Matrix coefficient is weight and importance between the number $i$ airport and the number $j$ factor by using the analytic hierarchy process.

In order to estimate the deviation of the weight in each airport matrix, need to calculate the 
similarity coefficient between the weights and composite the similarity coefficient matrix by using these similarity coefficient. Similarity coefficient $t_{i j}$ and similarity coefficient matrix $T$ is as follows:

$$
t_{i j}=1-\sqrt{\frac{1}{n} \sum_{k=1}^{n}\left(a_{i k}-a_{j k}\right)^{2}}
$$

$t_{i j}$ is the similarity between the weight of airport $i$ and airport $j$, the smaller the $t_{i j}$, the smaller the degree of similarity. $n$ is the dimension of the weight, represents the number of evaluation index. $n$ is the total number of airports, that is, the total number of airports to participate in the assessment of the weights. Obviously, $t_{i i}=1, t_{i j}=t_{j i}$ 。 Similarity coefficient matrix of ten airports as follows:

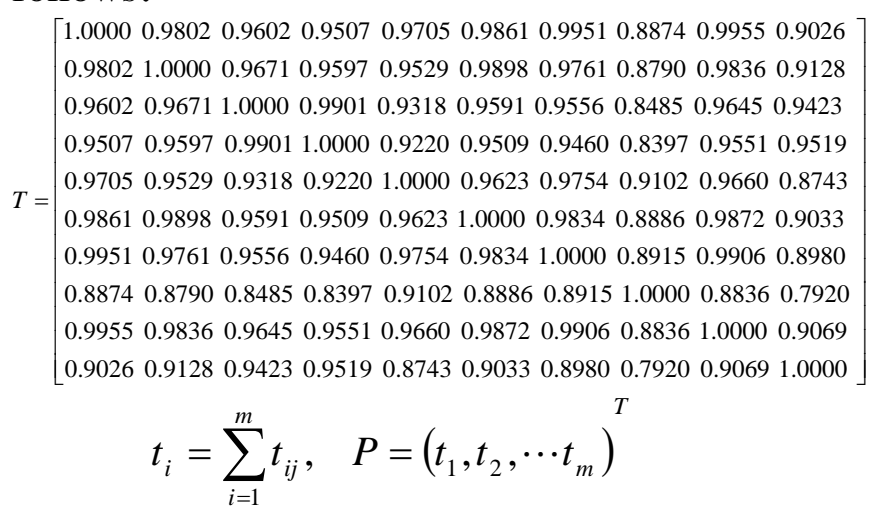

$t_{i}$ is the sum of the number in the line $i$ of similarity coefficient matrix, it represents the evaluation deviation degree between the weight of the number $i$ airport and other airports (including himself). The smaller the sum of similarity coefficient is, the more distant between airports is, and the greater the degree of deviation. " $P$ "indicates a sequence of similarity coefficient which the sum of the rows.The deviation degree of the ten airports is obtained as:

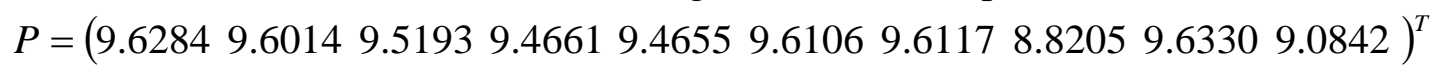

Eliminating the weight of a large degree deviation. This paper according to the expert eliminated rate eliminates the weight of a large degree deviation. In the process of elimination ,if the elimination of experts is too much, it will lost the role of group decision-making evaluation; if elimination number of the experts are too small, then it will make the expert who has large deviation degree had great influence on the evaluation results. According to the evaluation, it is more appropriate that the elimination rate of experts is in the $20 \% \sim 30 \%$. According to the eliminated rate standard of table 1, the weight provided by the no. 8 and no.10 airports can be eliminated, and average to the rest of the weight, the weight after the correction is:

$$
w=\left(\begin{array}{lll}
0.0968 & 0.2507 & 0.6525
\end{array}\right)
$$

\section{Summary}

Based on the adjusted weight $w$ and compared each delay percentage $Y$ between the project tier(sixty major international airports) in appendix 1 and standard tier, calculating the total weighted delay rate of main International Airport, and get the final ranking of the airport:

$$
T=w \times Y
$$

So we can get the consosulsion airports for flight delays are Xiaoshan Hangzhou, Pudong Shanghai, Hongqiao Shanghai, Baoan Shenzhen, Baiyun Guangzhou, O'Hare Chicago, LaGuardia New York, Xianyang Xi'an, Philadelphia, Jiangbei Chongqing.

\section{References}

[1]Tongke Shuang, Jialin Tian, et al. A method for determining index weight based on improved 
AHP. Science and technology in Western China. 2011(10) No.1,p.37-38.

[2] Information on: www.flightstats.com/go/Home/home.do.

[3] Information on: www.chinayearbook.com/yearbook/item/1/195639.html

[4]Qiyan Jiang, Jinxing Xie, et al. Mathematical Model [M]. Higher Education Press, 2014 year, p. 156-175. 